\title{
Tratamiento con endoprótesis y espirales de un pseudoaneurisma asociado a una estenosis en la arteria carótida interna
}

\author{
E. Górriz-Gómez; J.M. Carreira*; A. González García** y A. Mayo1-Deyá**
}

Unidad de Radiología Vascular Intervencionista. Hospital Universitario Nuestra Señora del Pino. Las Palmas de Gran Canaria. *Departamento de Radiología, Universidad de Santiago de Compostela. C.H.U.S. ** Neurorradiología Intervencionista. Hospital Universitario Virgen del Rocio. Sevilla.

\section{Resumen}

Propósito. Comunicar nuestra experiencia en el tratamiento de un paciente con un pseudoaneurisma asociado a una estenosis post-disección en la arteria carótida interna cervical.

Caso clínico. Paciente de 45 años valorado en el servicio de urgencias por hemiparesia izquierda. Entre sus antecedentes destacaba ser fumador importante, hipertensión arterial, hipercolesterolemia, peritonitis a los 18 años e infarto de miocardio hace un año. Tras la realización de TC y RM, en una angiografía cerebral se diagnosticó una oclusión de la arteria carótida interna derecha, presentando en la izquierda una estenosis asociada a un pseudoaneurisma. Mediante abordaje femoral común derecho, se realizó un implante de una endoprótesis metálica en la zona estenótica; a través de la malla de la misma, se excluyó el pseudoaneurisma con espirales metálicos.

Conclusión. Este caso muestra la posibilidad de recuperar la luz de una zona estenótica de la arteria carótida interna con una endoprótesis metálica no cubierta y simultáneamente embolización a su través de una zona pseudoaneurismática sin riesgo de migración.

PALABRAS CLAVE: .Endoprótesis. Arteria carótida. Pseudoaneurisma. Embolización

Internal carotid artery pseudoaneurysm and stenosis. Treatment with stents and coils

\section{Summary}

Purpose. To describe the endovascular management of a patient with postdisection internal carotid pseudoaneurysm and stenosis.

Case report. A 45-year-old man presented with left hemyparesia; angiography showed right internal carotid artery occlusion, left carotid stenosis and pseu-

Recibido: 02-02-04. Aceptado: 11-02-05 doaneurysm. A metallic uncovered stent was implanted by means of a femoral approach. Coils were delivered throught the uncovered stent and the pseudoaneurysm was excluded.

Conclusion. The internal carotid percutaneous implantation of a carotid uncovered stent, and embolization throught stent in a patient with stenosis and pseudoaneurysm, appears to be a safe procedure without risk of coils migration.

KEY WORDS: Stent. Carotid artery. Pseudoaneurysm. Embolization.

\section{Introducción}

Durante los últimos años hemos asistido a la generalización del uso de las endoprótesis vasculares. El territorio arterial periférico y fundamentalmente el territorio iliaco sirvió para demostrar la utilidad y resultados obtenidos con estos dispositivos ${ }^{14}$. Paulatinamente se fueron expandiendo las indicaciones de las endoprótesis vasculares a otros territorios arteriales y venosos obteniéndose resultados variables dependiendo del sector tratado ${ }^{7}$.

La indicación fundamental de las endoprótesis fue la recuperación de la luz vascular previamente estenótica o incluso obstruida. El perfeccionamiento de los materiales ha permitido la exclusión de aneurismas en diversos territorios vasculares, incluso vasos de gran calibre como la aorta $^{11}$.

El sector carotídeo también se ha visto beneficiado por la puesta en práctica de esta tecnología, si bien, en este caso

Abreviaturas. ATP: angioplastia transluminal percutánea. CAVATAS II: carotyd and vertebral artery transluminal angioplasty study. CREST; carotid revascularization endarterectomy vs. stenting trial. EVA IIIs: endarterectomy vs. angioplasty in patients with symptomatic severe carotid stenosis. GDC: coils (espirales) eolectrolargables de Gugliemi. RM: resonáncia magnética. SPACE: stenting protected angioplasty versus carotid endarterectomy. TC: tomografia computarizada. UI: unidades internacionales. 
las limitaciones iniciales fueron mayores que en el sector periférico, dadas las posibles complicaciones embólicas en el territorio cerebral; este hecho condicionó una variante técnica del implante, la protección distal, que no se utiliza habitualmente en el sector periférico.

Presentamos un paciente con patología estenótica de la arteria carótida interna y pseudoaneurisma post-disección tratado mediante implante de una endoprótesis no cubierta y embolización con espirales.

\section{Caso clínico}

Paciente de 45 años valorado en septiembre del año 2000 por hemiparesia izquierda. Entre sus antecedentes destacaba tabaquismo, hipertensión arterial, hipercolesterolemia, peritonitis a los 18 años e infarto de miocardio en 1999. Tras la realización de TC y RM, en una angiografía cerebral se diagnosticó una oclusión de la arteria carótida interna derecha y estenosis del $60 \%$ asociada a pseudoaneurisma en el trayecto cervical de la izquierda (figs. 1, 2), con signos de disección en la RM.

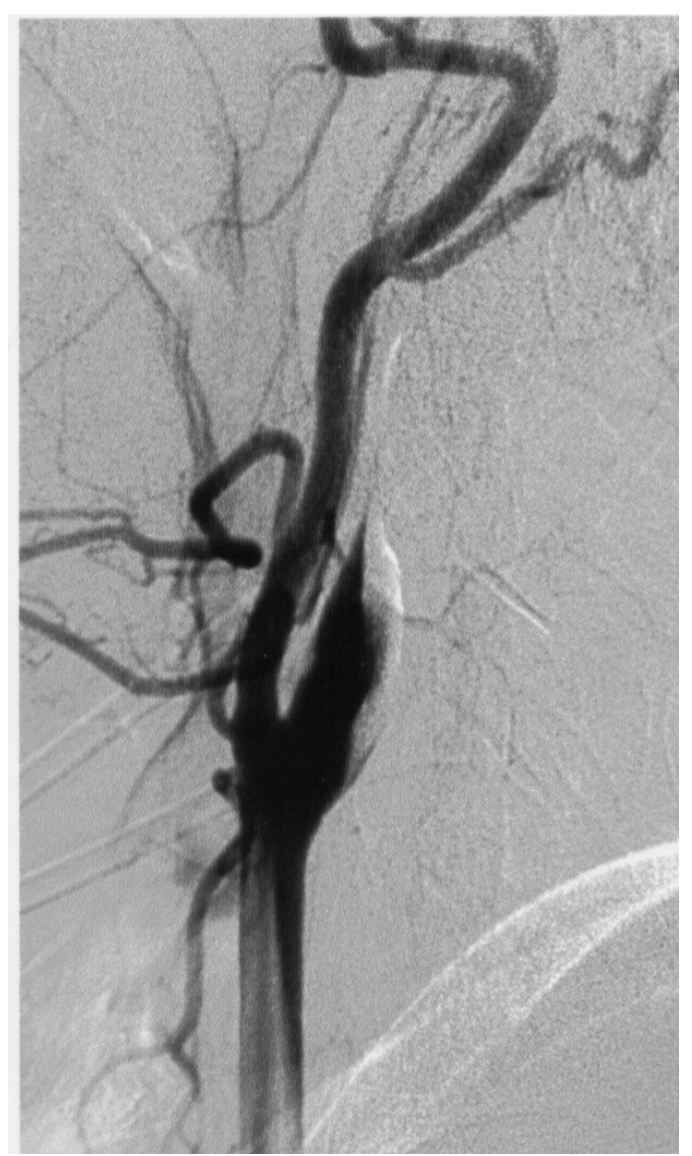

Figura 1. Una inyección de contraste en la arteria carótida primitiva derecha demuestra una obstrucción de la arteria carótida interna.

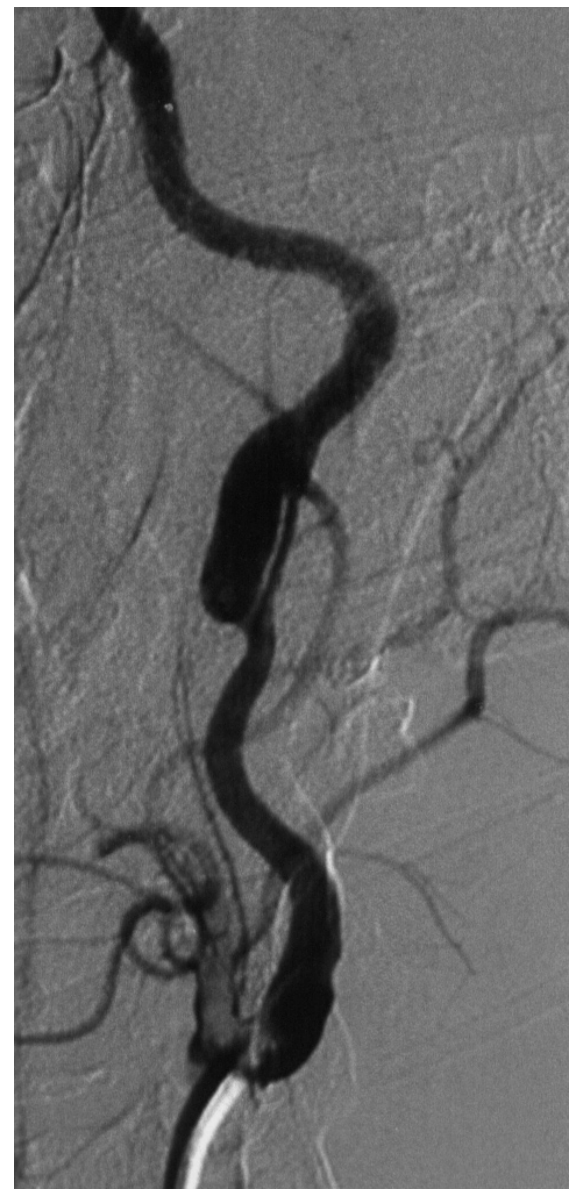

Figura 2. Una angiografía de la arteria carótida interna izquierda revela una estenosis y un pseudoaneurisma.

Se comprobó que había suplencia contralateral. Mediante abordaje femoral común derecho se realizó un implante de una endoprótesis metálica monorraíl (Carotid wallstent, Boston Scientific Co.) en la zona de la estenosis que se extendía también sobre la zona pseudoaneurismática del trayecto cervical de la arteria carótida interna izquierda (figs. 3, 4).

A través de la malla de la endoprótesis se introdujo un microcatéter con el que se liberaron espirales GDC (Boston Scientific Co.) sin riesgo de migración.

Un control angiográfico post-procedimiento evidenció la recuperación de la luz en la zona estenótica y la ausencia de relleno del pseudoaneurisma (fig. 5). Durante el procedimiento se administraron 5000UI de heparina sódica. Se realizó tratamiento anticoagulante secuencial en la fase aguda con heparina sódica en perfusión continua, seguido por dicumarinicos $\mathrm{y}$, posteriormente, antiagregantes plaquetarios.

Tras tres años de evolución, los controles angiográficos y ecográficos demostraron permeabilidad de la arteria tratada sin recidiva del pseudoaneurisma; el paciente no ha 
E. Górriz-Gómez y col

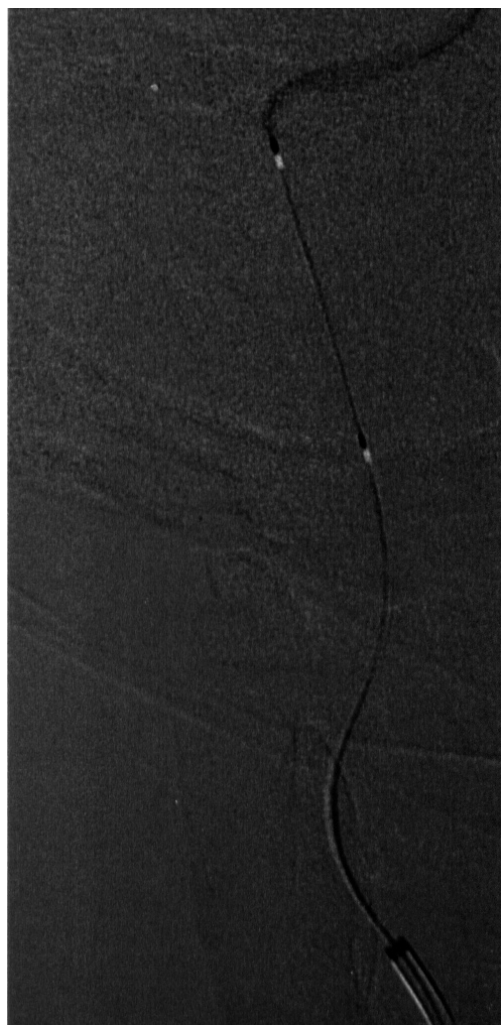

Figura 3. Posicionamiento de la prótesis previo a su liberación

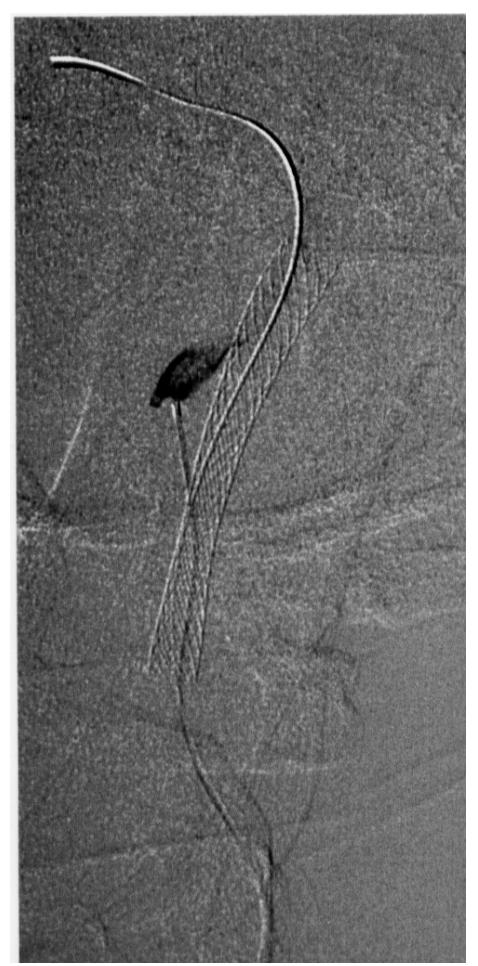

Figura 4. Tras la liberación de la endoprótesis metálica, se emboliza el pseudoaneurisma a través de la malla de la endoprótesis.
Neurocirugía $2005 ; 16: 528-532$

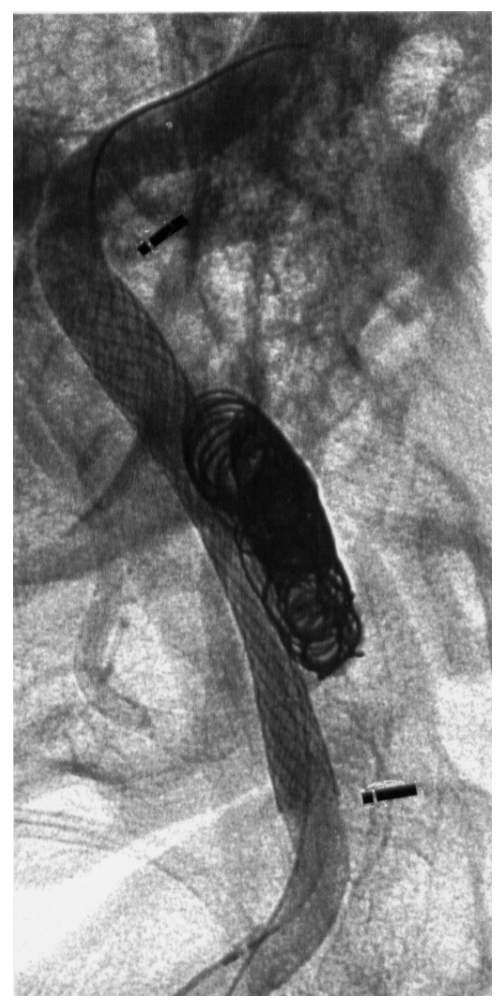

Figura 5. Un control angiográfico tras el procedimiento demuestra permeabilidad de la zona. Se aprecia contraste en la porción proximal y distal de la endoprótesis (flechas). Las espirales ocupan el pseudoaneurisma que no se rellena de contraste.

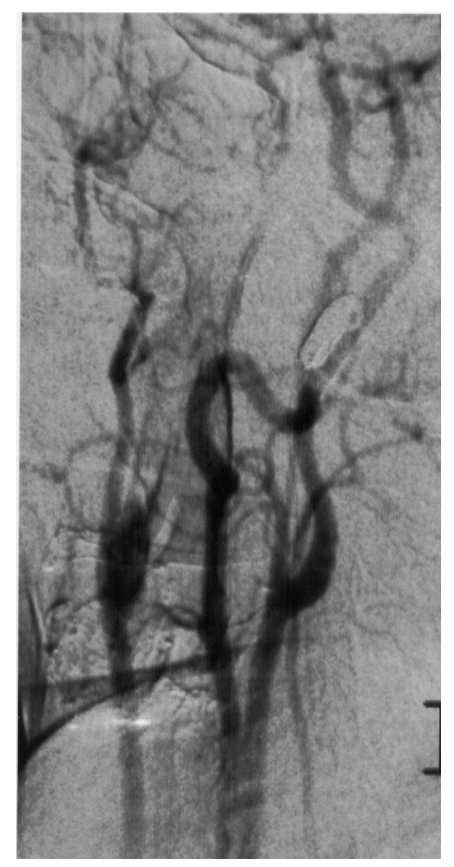

Figura 6.Angiografía realizada durante el seguimiento. El pseudoaneurisma no se rellena de contraste y la zona tratada permanece permeable. 
presentado síntomas neurológicos en territorio carotídeo izquierdo (fig. 6).

\section{Discusión}

La aplicación de la ATP en las lesiones estenóticas carotídeas supuso un cambio de la orientación terapéutica en este sector ${ }^{5} \mathrm{y}$ un paso previo a la utilización de endoprótesis vasculares en las lesiones estenóticas del sector carotídeo. Los resultados de estas endoprótesis están ampliamente probados a medio plazo aportando ventajas sobre el tratamiento quirúrgico convencional ${ }^{6}$. El perfeccionamiento de los materiales y de los mecanismos de protección y liberación hace que cada vez sea una práctica mas habitual, con índices de morbi-mortalidad bajos ${ }^{9}$.

El desarrollo de endoprótesis cubiertas, en el tratamiento de lesiones aneurismáticas en otros territorios anatómicos (aorta abdominal y torácica) ha aportado suficientes datos para tratar el mismo tipo de lesiones en el territorio carotídeo; sin embargo, estas prótesis, teóricamente ideales para el tratamiento del caso que nos ocupa, están en fase de desarrollo, intentando conseguir el mínimo diámetro del sistema de transporte y liberación $n^{4,17}$.

Recientemente se han publicado experiencias positivas que, con la utilización de estos dispositivos en lesiones aneurismáticas, obtienen buenos resultados iniciales ${ }^{10,13,17}$.

En la actualidad existen varios estudios en desarrollo para evaluar las indicaciones y resultados de las endoprótesis frente a endarterectomía en el territorio carotídeo (CREST, EVA-IIIs, CAVATAS II y SPACE), pero ya se han publicado series extensas ${ }^{5,6,8,12}$ y casos en indicaciones concretas ${ }^{10,15,17}$. Los resultados actuales indican que el porcentaje de éxitos y complicaciones puede ser similar entre la técnica con endoprótesis y la cirugía convencional, pero los resultados definitivos de los trabajos randomizados todavía no se conocen. Por otro lado, los resultados de estos estudios no reflejan la situación real de estas técnicas, ya que tanto endoprótesis nuevas como nuevos mecanismos de protección se han incorporado durante el desarrollo de estos estudios. De esta forma, en la práctica clínica la decisión depende de cada caso concreto y de las características de la lesión en cada paciente ${ }^{16}$.

En el caso que aquí describimos, se trata de un paciente con antecedentes neurológicos recientes, diagnosticado de obstrucción de la arteria carótida interna derecha y estenosis y pseudoaneurisma post-disección en la izquierda. En sesión clínica con el Departamento de Cirugía se decidió tratar ambas lesiones (siendo determinante eI grado de estenosis). Se deshechó el tratamiento quirúrgico y se valoró la posibilidad del tratamiento médico conservador o el percutáneo, decidiéndonos por este último por la edad, clínica y el grado de estenosis. Ante la imposibilidad de disponer de la endoprótesis cubierta, que hubiera solucio- nado ambos, se optó por el tratamiento secuencial, es decir, implantación de endoprótesis metálica convencional y relleno del saco aneurismático con espirales GDC.

La exclusión parcial del pseudoaneurisma con una prótesis permeable minimizó la posibilidad de migración de las espirales tras su liberación, permitiendo realizar un procedimiento seguro, lográndose simultáneamente la recuperación de la luz en la zona estenótica. Esta técnica permitió una liberación, sin riesgos de migración distal.

Tras tres años de seguimiento, el paciente no ha presentado síntomas neurológicos en territorio carotídeo izquierdo.

En dos trabajos previos, se describen casos similares al aquí descrito con un buen resultado a corto plazo ${ }^{1,3}$.

Creemos que este es un buen método para tratar simultáneamente una estenosis y un pseudoaneurisma carotídeo, minimizando los riesgos de migración. Hará falta mayor experiencia para valorar los resultados obtenidos en estos casos con prótesis cubiertas.

\section{Bibliografía}

1. Adel, M.M., Randall, T.H., Constantine, C.P. et al.: Dowd and Halbach. Endovascular Management of extracranial carotid artery dissection achieved using stent angioplasty. Am J Neuroradiol 2000; 21: 1280-1292.

2. Brophy, D.P., Hartnell, G.G., McEniff, N.J.: Percutaneous treatment of a symptomatic brachiocephalic artery stenosis with a Palmaz stent. Cardiovasc Intervent Radiol 1997; 20: 405-406.

3. Castro Reyes, E., Villoria Medina, F., Fortea Gil, F., Reparaz Asensio, L., Gil Nuñez, A.: Tratamiento reconstructivo de aneurisma de arteria carótida interna cervical mediante el uso combinado de endoprótesis autoexpandibles y coils tipo GDC. Técnicas endovasculares 2001; IV: 16-21.

4. Cwikiel, W., Harnek, J.: A new stent designed for carotid artery application. An experimental study. Acta Radiol 2000; 41: 601-604.

5. Gil-Peralta, A., Mayol, A., González Marcos, J.R. et al.: Percutaneous Transluminal Angioplasty of the Symptomatic Atherosclerotic Carotid Arteries. Results, complications and follow-up. Stroke 1996; 27: 2271-2273.

6. Guimaraens, L., Sola, M.T., Matali, A. et al.: Carotid angioplasty with cerebral protection and stenting: report of 164 patients (194 carotid percutaneous transluminal angioplasties). Cerebrovasc Dis 2002; 13: 114-119.

7. Kumar, K., Dorms, G., Bates, M.C., Palmer, L., Mathicak, L., Dufek, C.: Primary stent deployment in occlusive subclavian artery disease. Cathet Cardiovasc Diagn 1995; 34 : 281-285.

8. Maleux, G., Bernaerts, P., Thijs, V. et al.: Extracranial carotid artery stenting in surgically high-risk patients using the Carotid Wallstent endoprosthesis: midterm clinical and ultra- 
sound follow-up results. Cardiovasc Intervent Radiol 2003; 26: 340-346.

9. Martin, J.B., Pache, J.C., Treggiari-Venzi, M. et al.: Role of the distal balloon protection technique in the prevention of cerebral embolic events during carotid stent placement Stroke 2001; 32: 479-484.

10. Mukherjee, D., Roffi, M., Yadav, J.S.: Endovascular treatment of carotid artery aneurysms with stent grafts. J Invasive Cardiol 2002; 14: 269-272.

11. Parodi, J.C., Palmaz, J.C., Barone, H.D.: Transfemoral intraluminal graft implantation for abdominal aortic aneurysms. Ann Vasc Surg 1991; 5: 491-499.

12. Qureshi, A.I., Knape, C., Maroney, J., Suri, M.F., Hopkins, LN.: Multicenter clinical trial of the NexStent coiled sheet stent in the treatment of extracranial carotid artery stenosis: immediate and late clinical outcomes. J Neurosurg 2003; 99: 264-270.

13. Redekop, G., Marotta, T., Weill, A.: Treatment of traumatic aneurysms and arteriovenous fistulas of the skull base by using endovascular stents. J Neurosurg 2001; 95: 412-419.

14. Reyes, R., Maynar, M., Lopera, J. et al.: Treatment of Chronic Iliac Artery Occlusions with Guide Wire Recanaliza- tion and Primary Stent Placement. J Vasc Intery Radiol 1997; 8: 1049-1055.

15. Scavee, V., De Wispelaere, J.F., Mormont, E., Coulier, B., Trigaux, J.P., Schoevaerdts, J.C.: Pseudoaneurysm of the internal carotid artery: treatment with a covered stent. Cardiovasc Intervent Radiol 2001; 24: 283-285.

16. Sievert, H.: Carotid artery stenosis: who should undergo surgery and who should undergo stenting? J Intery Cardiol 2001; 14: 625-628.

17. Simionato, F., Righi, C., Melissano, G., Rolli, A., Chiesa, R., Scotti, G.: Stent-graft treatment of a common carotid artery pseudoaneurysm. J Endovasc Ther 2000; 7: 136-140.

Górriz-Gómez, E.; Carreira J.M.; González- García, A.; Mayol- Deyá, A.: Tratamiento con endoprótesis y espirales de un pseudoaneurisma asociado a una estenosis en la arteria carótida interna. Neurocirugía 2005; 16: 528-532.

Correspondencia postal: José M. Carreira. Facultad de Medicina. Departmento de Radiología. C/ San Francisco n 1. 15704 Santiago de Compostela. 